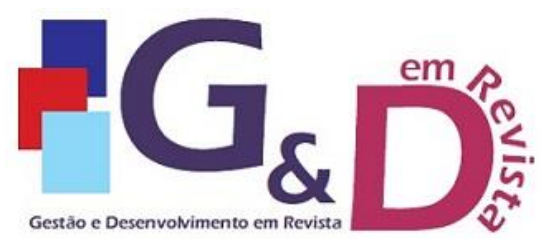

Gestão e Desenvolvimento em Revista

V. 8, N. 2, jul-dez/2021, p. 04-21.

ISSN online: $2446-8738$

Artigo recebido em: 28/10/2021

Artigo aprovado em: 28/11/2021

\title{
HEDGE ESTÁTICO E DINÂMICO PARA CONTRATOS FUTUROS DO BOI GORDO NA B3: UMA ANÁLISE COMPARATIVA ${ }^{1}$
}

\author{
Marcio José Szpaki Zaparolli \\ Mestrando pelo Programa de pós-graduação em Economia da Universidade Estadual de Maringá \\ (PCE/UEM). \\ E-mail:marcio_zap@hotmail.com \\ Luiz Gustavo Soares da Silva \\ Mestre em Matemática Aplicada e Computacional pela Universidade Estadual de Londrina - UEL/ \\ PGMAC. \\ E-mail: pg402467@uem.br \\ Margarete Campos Vieira \\ Mestrando pelo Programa de pós-graduação em Economia da Universidade Estadual de Maringá \\ (PCE/UEM). Atualmente é Professora do Centro Universitário Cidade Verde - UniFCV. \\ E-mail: pg402469@uem.br \\ Julyerme Matheus Tonin \\ Doutor em Economia Aplicada pela Escola Superior de Agricultura Luiz de Queiroz. Atualmente é \\ Professor Associado do Departamento de Economia da Universidade Estadual de Maringá - UEM. \\ E-mail: jmtonin@uem.br
}

\section{Resumo}

Tendo em vista a flutuação de preço no mercado e o longo período de maturação do investimento em gado de corte, é de interesse do agente racional tomar decisões que mitiguem eventuais perdas associadas a eventos não previstos. Dessa forma, o presente estudo busca analisar a efetividade da estratégia de hedge em contratos futuros de boi gordo, negociados na B3, como mecanismo financeiro de proteção ao risco de preço para os produtores de boi gordo, comparando as razões ótimas de hedge estático obtido por um modelo Vetorial de Correção de Erro - VEC com a razão ótima de hedge dinâmico obtida via modelo GARCH BEKK. O período da análise se estende de 2015 a 2020 e considera os preços no mercado doméstico. No estudo, foi constatado que a efetividade obtida no modelo dinâmico supera o modelo estático em poucas casas decimais. Apesar disso, em média a razão ótima de hedge dinâmico é menor que o estático.

Palavras-chave: Efetividade; Razão ótima de hedge; modelo VEC; modelo GARCH BEKK.

\begin{abstract}
Considering the market price fluctuation and the longtime of investment maturation in beff cattle, making decisions, which mitigate any losses associated to unpredictable events, is important by the rational agent. Thus, this study aims to analyze the hedge effectiveness strategy in live cattle future contracts negotiated on B3, as a financial mechanism to protect the price risk for beef producers. Optimal static hedge ratios obtained by Vector Error Correction - VEC static model were compared to the GARCH-BEKK model dynamic ratio. The effectiveness obtained by dynamic model barely overcomes the static model, for analyses period from 2015 to 2020, considering domestic prices. Although on average, the dynamic hedge optimal ratio is smaller than the static one.
\end{abstract}

Keywords: Effectiveness; Optimal hedge ratio; VEC model; GARCH BEKK model.

\footnotetext{
${ }^{1}$ Artigo apresentado no IX Congresso Nacional de Pesquisa em Ciências Sociais Aplicadas - Conape e publicado nos anais do respectivo Congresso e aprovado para publicação nessa Revista pelo sistema Fast-Track.
} 


\section{INTRODUÇÃO}

O Brasil possui um dos maiores rebanhos bovino do mundo, aproximadamente 215 milhões de cabeças, conforme a Pesquisa Pecuária Municipal de 2019 (IBGE, 2021), é líder mundial na exportação de carne bovina desde 2017 (USDA, 2020), dessa forma, a produção de carne bovina brasileira configura-se como um dos principais pilares do agronegócio. Segundo dados do Ministério da Indústria, Comércio Exterior e Serviços, as exportações de carnes e derivados bovinos $^{2}$ totalizaram cerca de US $\$ 8,5$ bilhões, o que corresponde a $4 \%$ da Balança Comercial Brasileira de 2020, um crescimento de 11,1\% em relação a 2019 . A China foi o principal destino da carne bovina brasileira, sendo responsável por $47,6 \%$ das vendas em dólares, seguido de Hong Kong (13,1\%), Egito (4,9\%) e Estados Unidos com 4,9\% (COMEX STAT, 2021).

Diante de magnitude e importância da produção bovina, como os produtores podem se proteger, de forma eficiente, da recorrente oscilação de preços no mercado, considerando o longo período de maturação do investimento em boi gordo? Isso posto, o presente trabalho busca analisar a efetividade da operação de hedge como uma solução fornecida pelo mercado futuro, na proteção do risco de preço para o boi gordo, aplicando o modelo estático e o dinâmico, afim de descobrir qual desses representa a melhor estratégia de proteção para o período de 2015 a 2020.

A atividade do hedger consiste em adotar uma posição contrária no mercado futuro à assumida no mercado à vista (HULL, 2016). Por exemplo, um produtor de gado de corte, para se proteger de uma queda no preço da commodity até sua data de venda, assume uma posição vendida em contrato futuro de boi gordo, obtendo receita nesse mercado caso o preço venha a cair, o que compensaria, se não totalmente, ao menos em parte, a perda que terá com a venda no mercado à vista a um menor preço, na data futura. No mercado financeiro o hedge é uma forma de proteção para o investimento que está exposto à volatilidade nos preços. Esse mecanismo funciona como uma trava de preços, cuja efetividade dependerá da razão do valor da posição assumida no mercado futuro em relação ao valor negociado no mercado à vista (HULL, 2016).

Diversas metodologias estudam a razão ótima de hedge. No presente trabalho, a metodologia adotada é a comparação entre a razão ótima estática obtida via maximização de uma função de utilidade do tipo média-variância, como abordado por Myers e Thompson (1989), com a razão ótima dinâmica estimada por um modelo GARCH-BEKK para a volatilidade da série dos retornos de um portfólio hedgeado, com a finalidade de descobrir qual modelo possui maior capacidade de proteção contra o risco de preço. Myers e Thompson (1989) explicam que o maior problema que enfrentam os comerciantes de commodities é saber qual o tamanho da posição que deve ser vendida no mercado futuro em relação a posição assumida no mercado à vista, essa proporção fornecerá a razão ótima de hedge que dependerá da variância dos preços nesses mercados.

A análise é semanal e se estende do início de 2015 ao final de 2020, período que se inicia com a quebra do ciclo de crescimento das exportações de carne bovina e, posteriormente, marca a retomada das mesmas, com o Brasil atingindo a marca

2 Refere-se ao critério adotado pela Associação Brasileira de Frigoríficos (ABRAFRIGO). O conjunto de NCMs que compõem a pauta exportadora pode ser consultado nas estatísticas disponíveis em seu site <http://www.abrafrigo.com.br/index.php/estatisticas/>. Acesso em 09 jan. 2021. 
de maior exportador mundial de carne bovina em 2017 (USDA, 2020). Utilizou-se o preço à vista no mercado físico para o estado de São Paulo, fornecido pelo Centro de Estudos Avançados em Economia Aplicada da Universidade de São Paulo (Cepea/USP), e a cotação do contrato futuro do boi gordo (BGI) negociado na bolsa brasileira Brasil, Bolsa, Balcão (B3).

\section{REFERENCIAL TEÓRICO}

Ao longo dos anos diversos estudos e teorias foram utilizadas para testar e otimizar a razão ótima de hedge. Conforme explica Rodrigues e Alves (2010), um dos autores mais influentes no século XX foi Holbrook Working, criando as bases para que Johnson (1960) e Stein (1961) desenvolvessem os conceitos matemáticos sobre a razão ótima de hedge, aplicando os conceitos da teoria do portfólio. O trabalho de Working também foi estudado por Ederington (1979) que analisou as três principais teorias de hedge da época: teoria tradicional, teoria de Holbrook Working e a teoria do portfólio. Ederington (1979) explicou que a decisão de se fazer hedge não difere das outras decisões de investimentos, no que diz respeito à busca pela melhor combinação entre risco e retorno.

A ideia básica por trás da teoria do portfólio é que os agentes buscam maximizar o retorno do seu portfólio minimizando os riscos, ou seja, minimizando a variância de sua carteira. No caso de uma operação com hedge, é possível encontrar a razão ótima a ser assumida no mercado futuro, em relação à posição à vista, que minimizará o risco da operação (a variância do retorno). Para isso, Johnson (1960) explicou que a receita obtida em um hedge de venda é dada da seguinte forma:

$$
R=x_{i} B_{i}+x_{j} B_{j}
$$

em que $B_{i}$ e $B_{j}$ representam a variação dos preços nos mercados $i$ e $j$ no período de tempo $t_{1}$ e $t_{2}, x_{i}$ e $x_{j}$ denotam o tamanho da posição assumida nos mercados $i$ e $j$. Seja $i$ o mercado à vista, denotado por $S$; e $j$ o mercado futuro, denotado por $F$, é possível rearranjar a equação anterior da seguinte forma:

$$
R=x_{i}\left(S_{2}-S_{1}\right)-x_{j}\left(F_{2}-F_{1}\right)
$$

Considerando que as posições assumidas em ambos mercados sejam de mesmo tamanho $\left(x_{i}=x_{j}\right)$, Johnson (1960) concluiu que um hedge é perfeitamente efetivo quando sua receita for nula, ou seja, $\left[\left(S_{2}-S_{1}\right)-\left(F_{2}-F_{1}\right)\right]=0$. Isso posto, ao se aplicar a variância na equação (1) tem-se:

$$
\begin{aligned}
& \operatorname{var}(R)=\operatorname{var}\left(x_{i} B_{i}+x_{j} B_{j}\right) \\
& \operatorname{var}(R)=x_{i}^{2} \operatorname{var}\left(B_{i}\right)+x_{j}^{2} \operatorname{var}\left(B_{j}\right)-2 x_{i} x_{j} \operatorname{cov}\left(B_{i}, B_{j}\right) \\
& \sigma_{R}^{2}=x_{i}^{2} \sigma_{i}^{2}+x_{j}^{2} \sigma_{j}^{2}-2 x_{i} x_{j} \operatorname{cov}_{i j}
\end{aligned}
$$




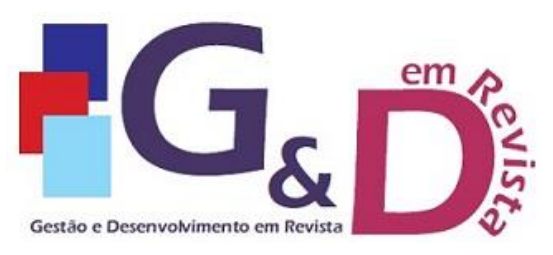

Gestão e Desenvolvimento em Revista V. 8, N. 2, jul-dez/2021, p. 04-21.

ISSN online: $2446-8738$

Artigo recebido em: 28/10/2021

Artigo aprovado em: 28/11/2021

Considerando que o problema consiste em decidir quais devem ser as quantidades escolhidas $\left(x_{i}, x_{j}\right)$ com o objetivo de minimizar a variância do retorno, temos o seguinte problema de otimização:

$$
\min _{\left(x_{i}, x_{j}\right)} \operatorname{var}(R)
$$

que é resolvido diferenciando a equação (3) em relação à $x_{i}$ e igualando a derivada a zero. Obtém-se o valor de $x_{j}$ que minimiza a variância da receita para $x_{i}, x_{j}^{*}$, uma vez que a derivada segunda é $2 \sigma_{j}^{2}>0$.

$$
\begin{aligned}
& \frac{\partial \sigma_{R}^{2}}{\partial x_{j}}=2 x_{j} \sigma_{j}^{2}-2 x_{i} \operatorname{cov}_{i j}=0 \\
& x_{j}=\frac{x_{i} \operatorname{cov}_{i j}}{\sigma_{j}^{2}}
\end{aligned}
$$

Sabendo que a razão ótima de hedge $h^{*}$ denota o tamanho da posição assumida no mercado futuro em relação à posição no mercado à vista $\left(x_{j} / x_{i}\right)$, ou seja, quanto do ativo físico é preciso proteger no mercado futuro para maximizar o retorno e minimizar o risco. Assumindo a hipótese que as séries de preços possuem variâncias e correlação constantes, é possível rearranjar a equação (4) para se obter $h^{*}$, que é dado pela seguinte expressão:

$$
h^{*}=\frac{x_{j}}{x_{i}}=\frac{\operatorname{cov}_{i j}}{\sigma_{j}^{2}}
$$

De posse da razão ótima de hedge, é possível calcular sua efetividade a partir da aplicação da equação (4) em (3), obtendo a seguinte expressão:

$$
\sigma_{R}^{2}=x_{i}^{2}\left[\sigma_{i}^{2}-\frac{\left(\operatorname{cov}_{i j}\right)^{2}}{\sigma_{j}^{2}}\right]
$$

Considerando $\rho$ como o coeficiente de correlação linear de $i$ e $j$, o mesmo é igual $\operatorname{acov}_{i j} / \sigma_{i} \sigma_{j}$. Portanto, a equação 6 pode ser expressa como:

$$
\sigma_{R}^{2}=x_{i}^{2} \sigma_{i}^{2}\left[1-\rho^{2}\right]
$$

Johnson (1960) explicou que quanto maior for o coeficiente de correlação, maior é a redução do risco de preço de se possuir o ativo $x_{i}$ dado a realização do hedge $x_{j}^{*}$. Portanto, a efetividade do hedge (e) é a proporção da variância da receita do hedge $\left(\sigma_{R}^{2}\right)$, associada à combinação $x_{i}, x_{j}^{*}$, em proporção à variância da receita $x_{i}^{2} \sigma_{i}^{2}$ de um portfólio sem hedge. Isto é: 


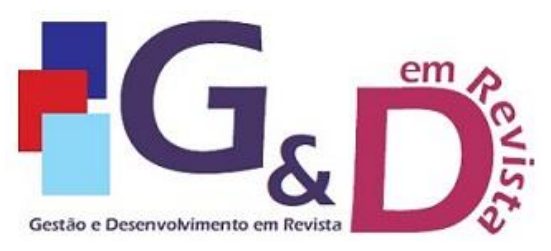

Gestão e Desenvolvimento em Revista V. 8, N. 2, jul-dez/2021, p. 04-21.

ISSN online: $2446-8738$

Artigo recebido em: 28/10/2021

Artigo aprovado em: 28/11/2021

$$
e=\left[1-\frac{\sigma_{R}^{2}}{x_{i}^{2} \sigma_{i}^{2}}\right]=\rho^{2}
$$

Dessa forma, quando utilizada a razão ótima de hedge, sua efetividade é igual ao quadrado do coeficiente de correlação linear dos preços no mercado à vista e futuro. Portanto, se os movimentos nos preços em $i$ e $j$ são iguais, a perda em um desses mercados é compensada integralmente por um ganho no outro, uma correlação perfeita entre ambos $(\rho=1)$ a qual o risco de preço é zero e o hedge considerado perfeitamente efetivo. Esta é uma situação ideal e distante do mundo real, porém, o que se conclui é que quanto maior for a correlação entre as variações nos preços à vista e futuro, mais efetivo é o hedge.

Diversos trabalhos aplicaram essa metodologia ao mercado de commodities na busca pela otimização das suas carteiras. Freitas e Alves (2013) estimaram o risco de base, a efetividade, e a razão ótima de hedge do boi gordo e cross-hedge do bezerro em contrato futuro de boi gordo para o estado de São Paulo no período de 2001 a 2010. Os autores mostraram que a razão ótima é maior para o boi gordo, necessitando uma maior quantidade de operações no mercado futuro para este, e a efetividade das operações de hedge foi cerca de $70 \%$ e $14 \%$, para o boi gordo e bezerro, respectivamente. Ou seja, para os produtores de boi, a estratégia de hedge consiste em uma boa ferramenta na proteção contra o risco de preço.

Tonin e Alves (2005) estudaram a efetividade e razão ótima de hedge dos contratos futuros de milho para a região de Maringá. Utilizaram modelos baseados na teoria do portfólio para determinar a receita de um hedger, além de terem utilizado relações de covariância para encontrarem a razão ótima de hedge.

Alves e Parré (2005) analisaram a cointegração, causalidade e efetividade do hedge para os preços do contrato de boi gordo no noroeste do Paraná. Demonstraram que a efetividade foi baixa devido à pouca liquidez no mercado, entre outros. Apesar disso, destacaram que o hedge é o mecanismo mais viável para fornecer aos integrantes da cadeia bovina a garantia de risco mínimo. Seus resultados mostraram um equilíbrio de longo prazo entre as variáveis, comprovando que elas se relacionam. Além disso, entre as variáveis preço futuro e preço à vista, verificou-se uma relação de bicausalidade mostrando que os agentes, ao tomarem suas decisões no mercado à vista, levam em consideração o preço de fechamento do dia anterior do contrato de primeiro vencimento, no mercado futuro; e no mercado futuro levam em consideração o que está ocorrendo no mercado à vista.

\section{METODOLOGIA}

\subsection{HEDGE ÓTIMO ESTÁTICO}

A abordagem empírica convencional para a estimação da razão ótima de hedge, proposta por Ederington (1979) é utilizar MQO no seguinte modelo:

$$
P_{v, t}=\beta_{0}+\beta_{1} P_{f, t}+u_{t}
$$

em que $u_{t}$ é um termo de erro estocástico, e o coeficiente angular da regressão acima é a estimativa de $h^{*}$. Um dos problemas desse método é o fenômeno da 
regressão espúria. A utilização de uma regressão em diferenças, isto é, em termos de retornos é o passo natural para uma tentativa de eliminar o fenômeno de regressão espúria. Conforme Rodrigues e Alves (2010) tal abordagem não será apropriada caso se verifique autocorrelação nos resíduos. Como proposta para solucionar o problema, Myers e Thompson (1989) apresentam uma família de modelos generalizados:

$$
P_{v, t}=\alpha_{0}+\delta P_{f, t}+\sum_{i=1}^{n} \beta_{i} P_{v, t-i}+\sum_{j=1}^{m} \gamma_{j} P_{f, t-j}+\sum_{k=1}^{q} \varphi_{k} X_{t-k}+u_{t}
$$

ou em diferenças:

$$
\Delta P_{v, t}=\alpha_{0}+\delta \Delta P_{f, t}+\sum_{i=1}^{n} \beta_{i} \Delta P_{v, t-i}+\sum_{j=1}^{m} \gamma_{j} \Delta P_{f, t-j}+\sum_{k=1}^{q} \varphi_{k} \Delta X_{t-k}+u_{t}
$$

em que: $\Delta$ é o operador de primeira diferença, $X_{k}$ são variáveis exógenas que ajudam explicar o comportamento dos preços e $n, m$ e $q$ são as defasagens adotadas segundo algum critério de informação. Nestes modelos $\delta$ é tomado como razão ótima de hedge.

Segundo Kumar (2008), uma maneira de se eliminar o problema de correlação serial entre os erros, e conjuntamente tornar a série de preços futuros endógena ao modelo, é estimar um modelo $\operatorname{VAR}(p)$ bivariado, que em diferenças apresenta a seguinte forma:

$$
\begin{aligned}
& \Delta P_{v, t}=\alpha_{01}+\sum_{i=1}^{p} \beta_{1 i} \Delta P_{v, t-i}+\sum_{j=1}^{p} \gamma_{1 j} \Delta P_{f, t-j}+e_{v, t} \\
& \Delta P_{f, t}=\alpha_{02}+\sum_{i=1}^{p} \beta_{2 i} \Delta P_{v, t-i}+\sum_{j=1}^{p} \gamma_{2 j} \Delta P_{f, t-j}+e_{f, t}
\end{aligned}
$$

em que, o vetor $\left(e_{v, t}, e_{f, t}\right)$ é um ruído branco bivariado.

Contudo, como sugere a hipótese de mercados eficientes, deve haver uma relação de equilíbrio de longo prazo entre os preços à vista e futuro de um mesmo ativo e, consequentemente, o retorno de um portfólio hedgeado deve ser um processo puramente aleatório, no sentido de que não seja possível operações com objetivo de arbitragem.

Sendo assim, Lien (1996) indica que se faz necessário a verificação se as séries de preços são processos cointegrados. Caso a resposta seja afirmativa, o modelo $\operatorname{VAR}(p)$ não é capaz de captar os efeitos no curto prazo de desvios do equilíbrio de longo prazo. O autor então sugere um modelo Vetorial de Correção de Erro - VEC $(p)$ como mais apropriado.

Considere o seguinte modelo $\operatorname{VEC}(p)$ em que, $Z_{t-1}$ é o termo de correção de erro, estimado como o resíduo da equação de cointegração $P_{v, t}=\varphi_{0}+\varphi_{1} P_{f, t}+u_{t}$ : 


$$
\begin{aligned}
& \Delta P_{v, t}=\alpha_{01}+\alpha_{v} Z_{t-1}+\sum_{i=1}^{p} \beta_{1 i} \Delta P_{v, t-i}+\sum_{j=1}^{p} \gamma_{1 j} \Delta P_{f, t-j}+e_{v, t} \\
& \Delta P_{f, t}=\alpha_{02}+\alpha_{f} Z_{t-1}+\sum_{i=1}^{p} \beta_{2 i} \Delta P_{v, t-i}+\sum_{j=1}^{p} \gamma_{2 j} \Delta P_{f, t-j}+e_{f, t}
\end{aligned}
$$

A ordem $p$ é determinada segundo algum critério de informação. Portanto, para esse modelo a razão ótima de hedge é:

$$
h^{*}=\frac{\sigma_{v, f}}{\sigma_{f}^{2}}
$$

$\operatorname{com} \sigma_{v, f}=\operatorname{cov}\left(e_{v, t}, e_{f, t}\right)$ e $\sigma_{f}^{2}=\operatorname{var}\left(e_{f, t}\right)$. Isso posto, o próximo subtópico abordará o modelo dinâmico e suas considerações.

\subsection{HEDGE ÓTIMO DINÂMICO}

Nos modelos estáticos, a persistência de heterocedasticidade nos resíduos ainda era um problema a ser tratado com o objetivo de proporcionar maior confiabilidade na estimativa da razão de hedge ótimo.

Conforme Myers e Thompson (1987), a abordagem correta é considerar que tanto os retornos esperados quanto as variâncias dos retornos devem ser condicionadas ao conjunto de informação disponível no momento. Os autores demonstram que, verificada a hipótese que os retornos no mercado futuro seguem um processo Martingale (mercado eficiente), a regra dada pela expressão (14) é a razão de hedge que maximiza uma utilidade do tipo média-variância. Com o surgimento dos modelos da família Generalized Auto-Regressive Conditional Heteroscedastic (GARCH) torna-se possível estimar as variâncias condicionadas. Adotando como hipótese de trabalho que os mercados sejam eficientes é possível estimar uma sequência $\left\{h_{t}^{*}\right\}$ de razões ótimas de hedge (dinâmicos) segundo a regra proposta por Myers e Thompson (1987).

Neste estudo o modelo heterocedástico condicional multivariado utilizado será o $\operatorname{BEKK}(1,1)$ com a dimensão $n=2$. Esta classe foi introduzida por Engle e Kroner (1995). O modelo a ser estimado é da forma:

$$
\Sigma_{t}=C C^{\prime}+A\left(\begin{array}{cc}
\varepsilon_{1, t-1}^{2} & \varepsilon_{1, t-1} \varepsilon_{2, t-1} \\
\varepsilon_{1, t-1} \varepsilon_{2, t-1} & \varepsilon_{2, t-1}^{2}
\end{array}\right) A^{\prime}+B \sum_{t-1} B^{\prime}
$$

Em que: $\Sigma_{t}$ é a matriz de covariâncias condicionada ao conjunto de todas as informações até o instante $t-1 ; A, B$ e $C$ são as matrizes $2 \times 2$ dos coeficientes a serem estimados, sendo $C$ uma matriz triangular inferior.

Nesse modelo, portanto, o número total de parâmetros a serem estimados é 11. Assume-se erros gaussianos e estima-se o modelo por meio do método da máxima verossimilhança. Estimado o modelo, as variâncias e covariâncias são geradas recursivamente através da expressão (15), que possibilitará o cálculo da razão ótima de hedge. 


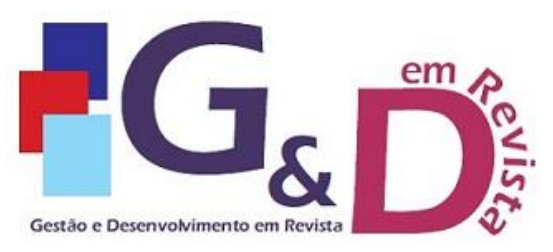

Gestão e Desenvolvimento em Revista V. 8, N. 2, jul-dez/2021, p. 04-21.

ISSN online: $2446-8738$

Artigo recebido em: 28/10/2021

Artigo aprovado em: 28/11/2021

$$
h_{t}^{*}=\left.\frac{\sigma_{v, f}}{\sigma_{f}^{2}}\right|_{t}
$$

Essa expressão demonstra o cálculo da razão de hedge ótimo dinâmico.

\section{ANÁLISE DOS DADOS}

Para a análise empírica, foram utilizados uma média diária ponderada de preços por arroba à vista de boi gordo no estado de São Paulo, elaborada pelo Centro de Estudos Avançados em Economia Aplicada da Universidade de São Paulo (Cepea/USP). Quanto aos preços no mercado futuro, referem-se ao contrato futuro do boi gordo (BGI), da série de primeiro vencimento em aberto, negociado na bolsa de valores brasileira Brasil, Bolsa, Balcão (B3), e cotados em reais por arroba líquida. Esses dados foram fornecidos pelo site Investing.com. O produto negociado por esses contratos são bovinos machos, com 16 arrobas líquidas ou mais, de carcaça e idade máxima de 42 meses (B3, 2021). Não são levados em conta os custos de transação sobre as operações: corretagem, taxas, tributos, etc.

O período analisado vai de 01 de janeiro de 2015 a 31 de dezembro de 2020, a frequência dos dados é semanal, em que se adotou a quarta-feira como referência para a determinada semana, dado a maior liquidez nas negociações em bolsa, contornando um provável efeito calendário do final de semana. Na ocorrência de feriados, utilizou-se a quinta-feira como substituto. Dessa forma, obtém-se um registro para cada semana do ano, totalizando 313 observações para todo o período de análise.

No presente estudo, define-se como notação a utilização de $P_{v}$ para a série de preços no mercado a vista e $P_{f}$ como a série de preços no mercado futuro. $\mathrm{Na}$ análise, será aplicado uma transformação logarítmica em ambas as séries, o que permite a identificação entre as primeiras diferenças e os retornos. As análises estatísticas foram feitas usando o software R.

As séries de preços transformadas são apresentadas no painel superior da Figura 1. Conforme a figura sugere, as séries de preços à vista e futuro para o boi gordo não parecem ser geradas por processos estocásticos estacionários, sugerindo uma possível cointegração.

Figura 1 - Série dos preços e retornos à vista e futuro.

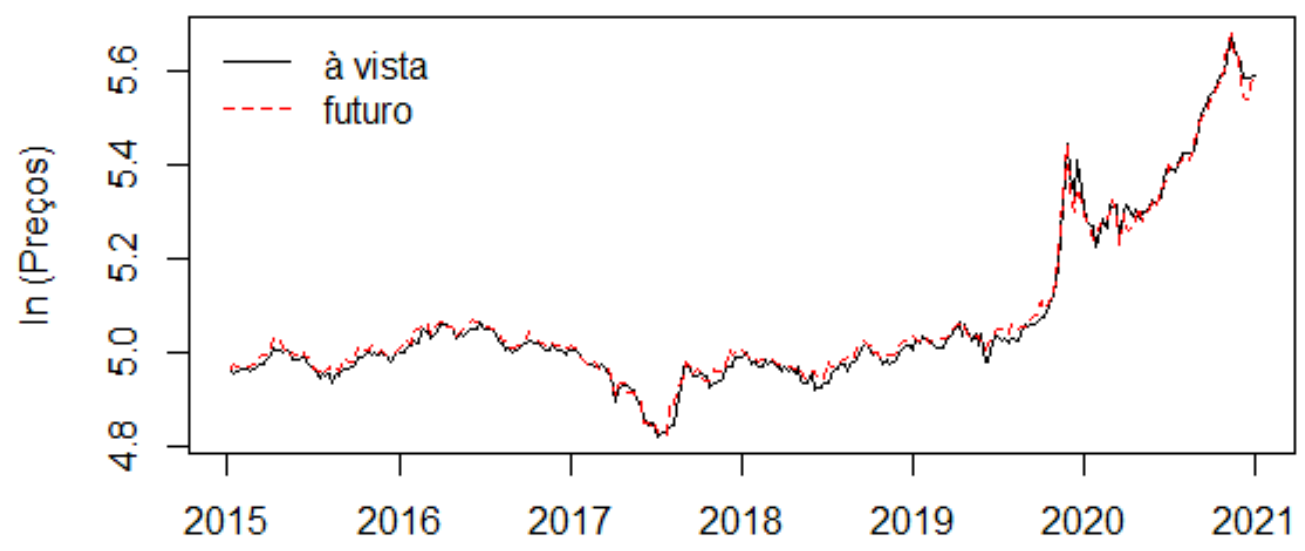




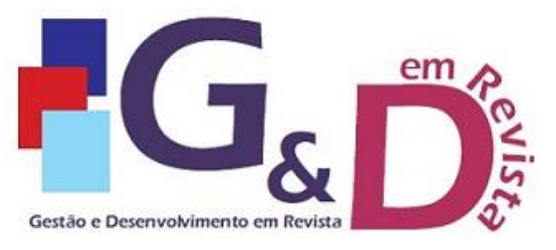

Gestão e Desenvolvimento em Revista V. 8, N. 2, jul-dez/2021, p. 04-21.

ISSN online: $2446-8738$

Artigo recebido em: 28/10/2021

Artigo aprovado em: 28/11/2021

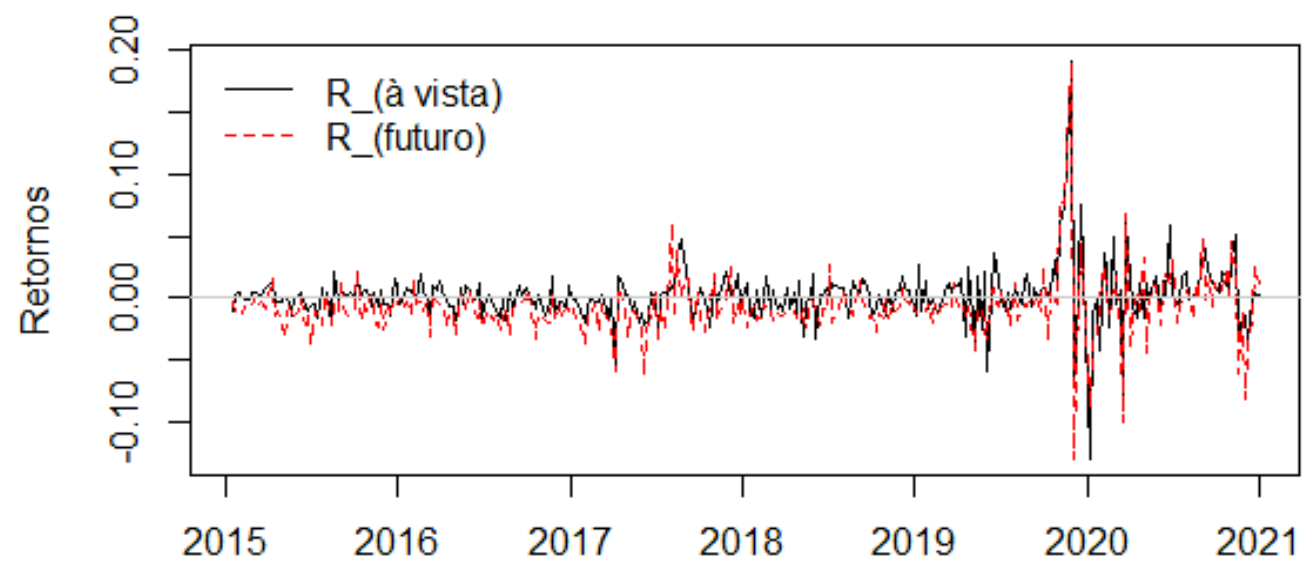

Fonte: Elaborado pelos autores (2021).

No painel inferior, observando-se o gráfico das primeiras diferenças, existe evidência da média dos processos serem centradas em zero, isto é, que as esperanças dos retornos sejam nulas. Contudo, em relação à variabilidade dos retornos, parece haver momentos de maior variabilidade (grupos de volatilidade), indicando forte indício de não estacionariedade nas variâncias.

Analisando a presença de raiz unitária, foi realizado o teste de Dickey-Fuller Aumentado (ADF) para as versões: sem constante, com constante, com constante e tendência; o número de defasagem dos modelos testados foi selecionado através do Critério de Informação de Akaike (AIC). Os resultados estão sintetizados na Tabela 1.

Tabela 1 - Testes de Raiz Unitária (lag = 1).

\begin{tabular}{cc|cc|cccc}
\hline \multirow{2}{*}{ Série } & \multicolumn{3}{c}{ Sem constante } & \multicolumn{2}{c}{ Com constante } & \multicolumn{2}{c}{$\begin{array}{c}\text { Com constante e } \\
\text { tendência }\end{array}$} \\
\cline { 2 - 7 } & t-ADF & $\begin{array}{c}\text { ADF } \\
\text { Crítico* }\end{array}$ & t-ADF & $\begin{array}{c}\text { ADF } \\
\text { Crítico* }\end{array}$ & t-ADF & $\begin{array}{c}\text { ADF } \\
\text { Crítico* }\end{array}$ \\
\hline $\ln \left(P_{v}\right)$ & 1.571 & -1.95 & 0.649 & -2.87 & -0.692 & -3.42 \\
$\ln \left(P_{f}\right)$ & 1.671 & -1.95 & 1.003 & -2.87 & -0.434 & -3.42 \\
$\Delta \ln \left(P_{v}\right)$ & -11.859 & -1.95 & -11.986 & -2.87 & -12.161 & -3.42 \\
$\Delta \ln \left(P_{f}\right)$ & -11.815 & -1.95 & -11.971 & -2.87 & -12.200 & -3.42 \\
\hline \multicolumn{5}{r}{ Fonte: Elaborado pelos autores $(2021)}$. \\
* Nível de Significância $=5 \%$ &
\end{tabular}

Considerando a hipótese nula do teste ADF como a não estacionariedade (presença de raíz unitária) contra a hipótese alternativa de estacionariedade, observa-se que não se deve rejeitar a não-estacionariedade das séries dos logaritmos dos preços à vista e futuro. Por outro lado, aceita-se a estacionariedade das séries dos retornos para ambos os mercados.

Os resultados acima também indicam que as séries são integradas de mesma ordem, ou seja, $\left\{\ln \left(P_{v}\right)\right\}_{t} \sim I(1)$ e $\left\{\ln \left(P_{f}\right)\right\}_{t} \sim I(1)$. Sendo assim, com base na teoria econômica, têm se por hipótese a existência de um equilíbrio de longo prazo entre o preço de um ativo no presente e seu preço no mercado futuro. Empiricamente, tal hipótese pode ser testada verificando se as séries compartilham da mesma tendência estocástica. Caso a resposta seja afirmativa, diz-se que as 
séries são cointegradas e, portanto, são válidas as condições do Teorema de Representação de Granger. Tal teorema diz, a grosso modo, que desequilíbrios de curto prazo são corrigidos para que a cointegração das séries seja observada. Neste contexto, conforme argumentado na parte teórica, fica justificado o uso de um VEC $(p)$ para estimativa da razão ótima de hedge estático.

Para a verificação da cointegração entre as séries de preços utilizou-se o teste de Phillips-Ouliaris que possui a seguinte hipótese nula a ser testada: $\mathrm{H}_{0}$ : As séries não são cointegradas. O teste foi implementado utilizando a função po.test contida na biblioteca tseries do software $\mathbf{R}$ e produziu um $\mathrm{p}$-valor $<0,01$. Sendo assim, rejeita-se a hipótese nula e considera-se neste trabalho que as séries dos logaritmos dos preços no mercado à vista e futuro são cointegradas de ordem 1 .

\section{RESULTADOS}

Nesta seção são apresentadas as estimativas da razão ótima de hedge conforme o tipo de modelagem, a saber: estático (VEC) e dinâmico (BEKK). Também são analisadas e comparadas as efetividades das estratégias de hedge adotadas.

\subsection{MODELO VEC E RAZÃO ÓTIMA DE HEDGE ESTÁTICO}

Com o objetivo de corroborar o resultado da seção anterior e estimar o modelo Vetorial de Correção de Erros (VEC), foi adotado o procedimento sugerido por Engle e Granger (1987) para se verificar a cointegração das séries. Isto é, estima-se a cointegração regredindo o preço à vista contra o preço futuro, considerando as séries em log, e verificando se os resíduos são estacionários. Os resultados podem ser observados na Tabela 2 .

Tabela 2 - Análise de resultados da regressão de cointegração.

\begin{tabular}{lcccc}
\hline \multicolumn{1}{c}{ Coeficiente } & Estimativa & Desvio Padrão & Teste t & p-valor \\
\hline Intercepto & -0.03745 & 0.02136 & -1.754 & 0.0805 \\
$\ln \left(P_{f}\right)$ & 1.00699 & 0.00421 & 239.217 & 0.0000 \\
\hline R2 & 0.9948 & & \\
$\mathrm{~F}$ & $5.722 \mathrm{e}+04$ & $\mathrm{gl}=(1.301)$ & 0.0000 \\
\hline \multicolumn{5}{c}{ Fonte: Elaborado pelos autores (2021). }
\end{tabular}

O teste de Raiz Unitária (ADF) para a séries dos resíduos da regressão de cointegração é fornecido pela Tabela 3. Como $t$ calculado é maior que o crítico em todas as opções, rejeita-se a hipótese nula da presença de raiz unitária nos resíduos da equação de cointegração, logo, a série é estacionária. O modelo $\operatorname{VEC}(p)$, conforme a equação (13), é estimado utilizando o método dos MQO em dois estágios, o número de defasagens do modelo utilizado para o cálculo da razão ótima de hedge é 1, conforme o menor critério de Akaike demonstrado pela Tabela 3 a seguir. 


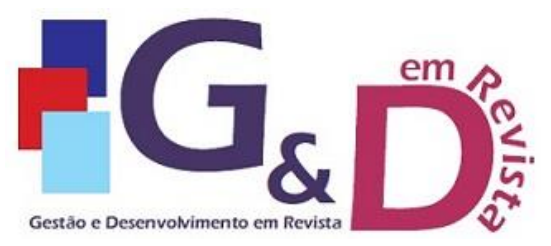

Gestão e Desenvolvimento em Revista V. 8, N. 2, jul-dez/2021, p. 04-21.

ISSN online: $2446-8738$

Artigo recebido em: 28/10/2021

Artigo aprovado em: 28/11/2021

Tabela 3 - Teste de Raiz Unitária dos resíduos da regressão de cointegração (lag = 1).

\begin{tabular}{|c|c|c|c|c|c|c|}
\hline \multirow{2}{*}{$\begin{array}{l}\text { Série dos } \\
\text { resíduos }\end{array}$} & \multicolumn{2}{|c|}{ Sem constante } & \multicolumn{2}{|c|}{ Com constante } & \multicolumn{2}{|c|}{$\begin{array}{c}\text { Com constante e } \\
\text { tendência }\end{array}$} \\
\hline & t-ADF & $\begin{array}{c}\text { ADF } \\
\text { Crítico* }\end{array}$ & t-ADF & $\begin{array}{c}\text { ADF } \\
\text { Crítico* }\end{array}$ & $\mathrm{t}-\mathrm{ADF}$ & \begin{tabular}{|c} 
ADF \\
Crítico*
\end{tabular} \\
\hline$\hat{u}_{t}$ & -8.8456 & -1.95 & -8.8308 & -2.87 & -8.8154 & -3.42 \\
\hline № de defasagens & 1 & & 2 & 3 & & 4 \\
\hline Critério Akaike & $-5003,88$ & & $-4979,97$ & $-4970,90$ & & $-4947,15$ \\
\hline
\end{tabular}

As estimativas para os coeficientes do modelo estão apresentadas na Tabela 4, o vetor de integração é fornecido pela Tabela 2. Cabe ressaltar que apenas o coeficiente do termo de correção de erro da equação que explica a primeira diferença do logaritmo do preço à vista, $\Delta \ln \left(P_{v, t}\right)$, é estatisticamente significante e possui o sinal esperado. Tal fato está de acordo com o critério de informação, que revelou que acréscimos no número de defasagens no modelo não ajudam na melhora da explicação das variáveis endógenas $\Delta \ln \left(P_{v}\right)$ e $\Delta \ln \left(P_{f}\right)$.

Tabela 4 - Estimativas dos coeficientes segundo modelo VEC(1).

\begin{tabular}{ccc}
\hline & $\Delta \ln \left(P_{v, t}\right)$ & $\Delta \ln \left(P_{f, t}\right)$ \\
\hline$\Delta \ln \left(P_{v, t-1}\right)$ & -0.0875 & 0.0345 \\
& $(0.0948)$ & $(0.1011)$ \\
$\Delta \ln \left(P_{t, t-1}\right)$ & 0.0601 & -0.0496 \\
& $(0.1079)$ & $(0.1150)$ \\
TCE & $-0.7173^{* * *}$ & -0.1220 \\
& $(0.1140)$ & $(0.1215)$ \\
\hline
\end{tabular}

Fonte: Elaborado pelos autores (2021).

*** Nível de Significância $<0,1 \%$

Obs: $O$ termo de correção de erro (TCE) é obtido pela seguinte expressão: $\ln \left(P_{v, t-1}\right)+0.03745-1.0069 \ln \left(P_{f, t-1}\right)$.

Em resumo, pode-se interpretar o modelo VEC(1) estimado da seguinte maneira: Primeiro, o coeficiente angular da regressão cointegrante é próxima a 1, indicando que mudanças dos preços do boi gordo à vista e futuro devem possuir aproximadamente a mesma magnitude para que se mantenha o equilíbrio de longo prazo entre esses mercados. Caso, por exemplo, exista um desequilíbrio no curto prazo onde o preço à vista exceda em $\delta>0$ unidades monetária o preço futuro, o retorno do mercado à vista deve cair aproximadamente 0,7173 para que se restabeleça o equilíbrio de longo prazo.

Uma rápida análise gráfica dos resíduos é fornecida pelas figuras A1 e A2, constantes no apêndice, a fim de demonstrar a característica de ruído branco. Também são apresentadas a plotagem da série dos resíduos, histograma, diagrama de dispersão e o gráfico da função de autocorrelação (ACF) na Figura A3.

Como pode-se observar no gráfico das séries dos resíduos na Figura $A 1$ e Figura A2, o modelo VEC(1) estimado não foi capaz de incorporar a volatilidade da série dos retornos. Contudo, os resíduos apresentaram alta correlação $\rho_{\varepsilon_{v}, \varepsilon_{t}}^{\text {vec }}=0,83$, 
com médias próximas de zero. A presença de heterocedasticidade condicional, então, pode ser evidenciada pelo gráfico da Função de Autocorrelação (ACF) dos quadrados desses resíduos, como mostra a Figura A3. Cabe ressaltar que tal análise não substitui um teste estatístico para detectar a presença de heterocedasticidade condicional.

Isso posto, uma vez obtidos os resíduos do modelo $\left(e_{v, t}, e_{t, t}\right)$, calcula-se a matriz de covariância para se obter a razão de hedge ótima estática $h^{*}=\sigma_{v, f} / \sigma_{f}^{2}$, encontrando o seguinte resultado:

$$
h_{\mathrm{vec}}^{*}=0,775 \text {. }
$$

Assim, a equação (17) demonstra que para o modelo estático a razão ótima a ser hedgeada equivale a cerca de $77,5 \%$ da exposição no mercado à vista (spot). Vejamos a seguir o modelo dinâmico.

\subsection{MODELO BEKK(1,1) E RAZÃO ÓTIMA DE HEDGE DINÂMICO}

O próximo passo é a estimação de um modelo $\operatorname{BEKK}(1,1)$ para se gerar matrizes de covariâncias para as séries dos retornos do boi gordo no mercado à vista e futuro. As matrizes dos coeficientes estimados por máxima verossimilhança são:

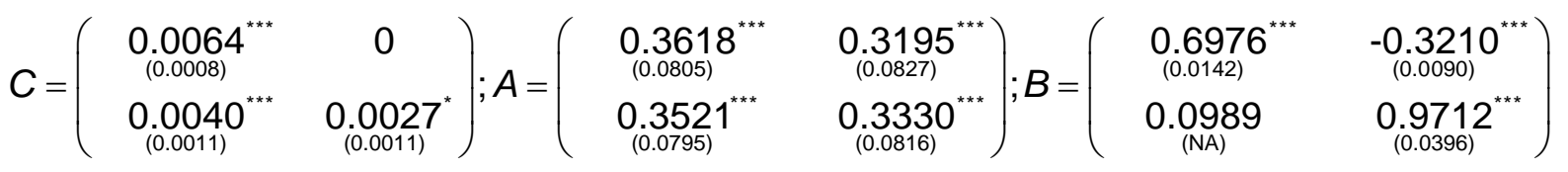

os asteriscos denotam o nível de significância: ${ }^{* *}<0.001 ;{ }^{* *}<0.01 ; \mathrm{e}^{*}<0.05$. Os valores entre parênteses são os respectivos desvios-padrão.

As matrizes de covariância são geradas recursivamente pela equação (15). O cálculo da razão ótima dinâmica é demonstrado na Figura 2, conjuntamente com a razão estática obtida na Seção 4.1.

Figura 2 - Razão ótima de hedge.

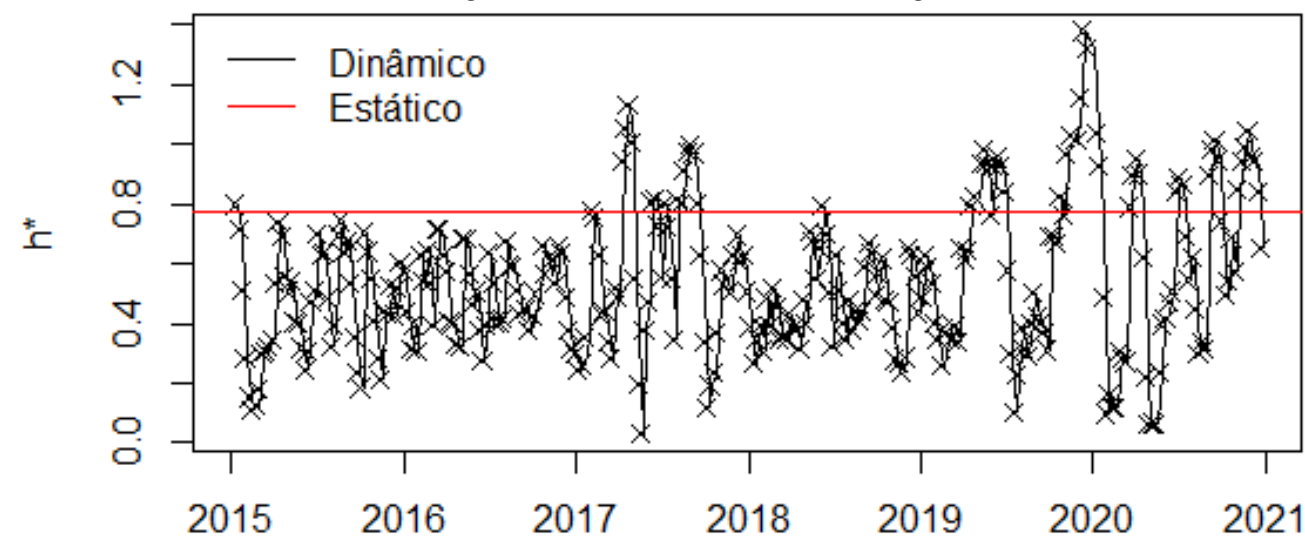

Fonte: Elaborado pelos autores (2021).

As estatísticas descritivas da razão dinâmica são resumidas na Tabela 5: 
Tabela 5 - Razão dinâmica.

\begin{tabular}{cccccc}
\hline Mínimo & $\mathbf{1}^{\circ} \mathbf{Q}$ & Mediana & Média & $\mathbf{3}^{\circ} \mathbf{Q}$ & Máximo \\
\hline 0.03 & 0.37 & 0.51 & 0.54 & 0.68 & 1.38 \\
\hline \multicolumn{7}{c}{ Fonte: Elaborado pelos autores (2021). }
\end{tabular}

Portanto, os resultados observados demonstram que no período da análise a estratégia de hedge dinâmico é flexível, sugerindo momentos de posicionamento relativamente baixo no mercado futuro $\left(\min h^{*}=0,03\right)$, e momentos de posição consideravelmente maior neste mercado, em relação ao mercado à vista (max $\left.h^{*}=1,38\right)$.

\subsection{EFETIVIDADE DAS ESTRATÉGIAS DE HEDGE}

O presente estudo tem por objetivo avaliar o desempenho de 4 estratégias de hedge para o mercado do boi gordo no período:

i. Exposição total $(h=0)$;

ii. Cobertura total ou naive hedge $(\mathrm{h}=1)$;

iii. Razão ótima estática;

iv. Razão ótima dinâmica.

O critério de desempenho de uma estratégia baseia-se no conceito de efetividade, isto é, o percentual de redução na variância de um portfólio hedgeado frente ao portfólio benchmark, que neste estudo será a ausência de hedge $(h=0)$. Para fins de comparação, também é adotada a estratégia ingênua de uma razão de hedge $h=1$, no sentido de ignorar o risco de base e operar em um mundo determinístico de hedge perfeito.

Considere a série de receitas obtida por uma estratégia de hedge $\left\{h_{t}\right\}$, em um dado período de tempo $t=1,2, \ldots, n$ :

$$
R_{t}^{h}=q_{v}\left(\Delta \ln P_{v, t}-h_{t} \Delta \ln P_{f, t}\right) .
$$

A efetividade de uma determinada estratégia de hedge $\tilde{h}$ é dada por:

$$
e=\frac{\operatorname{var}\left(R^{0}\right)-\operatorname{var}\left(R^{\tilde{n}}\right)}{\operatorname{var}\left(R^{0}\right)}
$$

sendo $R^{0}$ a estratégia benchmark e $R^{\tilde{h}}$ a estratégia adotada. 


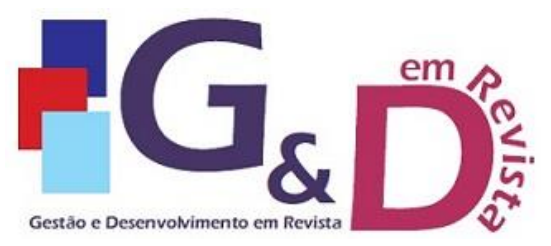

Gestão e Desenvolvimento em Revista

V. 8, N. 2, jul-dez/2021, p. 04-21.

ISSN online: $2446-8738$

Artigo recebido em: 28/10/2021

Artigo aprovado em: 28/11/2021

Tabela 6 - Efetividade por tipo de estratégia.

\begin{tabular}{lccc}
\hline \multicolumn{1}{c}{ Estratégia } & Desvio Padrão & Razão Ótima* $^{*}$ & Redução do Risco $^{*}$ \\
\hline Sem hedge & 0.022 & - & 0.00 \\
Naive hedge $^{* *}$ & 0.015 & 1 & 0.54 \\
Hedge Estático & 0.014 & 0.775 & 0.58 \\
Hedge Dinâmico & 0.013 & 0.54 & 0.61 \\
\hline
\end{tabular}

Fonte: Elaborado pelos autores (2021).

* Para a abordagem dinâmica é apresentada a razão ótima de hedge em termos médios.

** Naive hedge, ou cobertura ingênua é a estratégia em que o instrumento no mercado à vista é totalmente coberto com um instrumento futuro.

A Tabela 6 faz um apanhado das quatro estratégias analisadas e demonstra a efetividade da operação de hedge enquanto capacidade de reduzir o risco da operação. A estratégia com melhor resultado foi a de hedge dinâmico, em que $54 \%$ do valor dos ativos é hedgeado, reduzindo o risco da operação em $61 \%$.

\section{CONSIDERAÇÕES FINAIS}

$\mathrm{Na}$ gestão de risco de preço de commodities agropecuária, a implementação da operação de hedge com a utilização de contratos futuros é uma opção muito utilizada para mitigar o risco de preços. Sabendo-se que há diferentes abordagens teóricas para avaliar a efetividade e a razão ótima de uma estratégia de hedge, no presente estudo optou-se por realizar uma análise estática, conforme descrito por Myers e Thompson (1989), e uma abordagem dinâmica, por meio da estimação de um modelo GARCH-BEKK. O objeto de estudo foi o mercado futuro de boi gordo e o recorte temporal refere-se ao período de 2015 a 2020.

Dentre os achados de pesquisa, cabe destacar que a estratégia com maior efetividade na operação de hedge em contratos futuros de boi gordo é aquela obtida pelo modelo dinâmico BEKK $(1,1)$. Na comparação entre os modelos dinâmico e estático, observou-se que as efetividades foram de 0,61 e 0,58, respectivamente; as razões ótimas de hedge foram de 0,540 e 0,775, respectivamente.

Dito de outra forma, o que se conclui é que na estratégia estática, o agropecuarista deve vincular $77,5 \%$ do seu mercado físico (produção a ser comercializada) a contratos futuros na B3, a fim de reduzir o risco de preço em torno de $58 \%$. Para a estratégia dinâmica, a razão ótima a ser hedgeada é, em média, $54 \%$ da produção a ser comercializada, com redução de $61 \%$ do risco.

Segundo o modelo dinâmico, pode-se notar grande variabilidade na razão ótima ao longo do período analisado. Desse modo, a parcela do mercado físico atrelado a contratos futuros oscila, principalmente, entre $37 \%$ ( $1^{\circ}$ quartil) e $68 \%$ ( $3^{\circ}$ quartil), podendo elevar-se rapidamente em momentos de estresse no mercado.

Uma limitação do presente estudo é que o mesmo se limitou a comparar a razão ótima e a efetividade entre a utilização de abordagens estaticas e dinâmicas. Não foram levados em conta os custos presentes na rolagem de hedge, que ocorrem em uma estratégia dinâmica; bem como as dificuldades em termos de fluxos de caixa (necessidade de depósito de margens e ajustes diários), caso fosse necessário realizar uma grande mudança no volume de contratos futuros negociados.

Para futuros estudos, sugere-se a inclusão de variáveis exógenas como câmbio, e/ou, que estejam relacionadas com o movimento de oferta e demanda 
nesse mercado, na tentativa de ajudar na previsão dos movimentos dos preços, possibilitando uma maior acurácia na estimativa de suas correlações puras.

\section{REFERÊNCIAS}

AKAIKE, $\mathrm{H}$. Information theory and an extension of the maximum likelihood principle. In: PETROV, B. N.; CASKI, F. (Org.), 1973. Proceedings of the Second International Symposium on Information Theory. Budapest: Akademiai Kiado. p. 267-281, 1973.

AKAIKE, $\mathrm{H}$. A new look at the statistical model identification. IEEE Transactions on Automatic Control, v. 19, n. 6, p. 716-723, 1974.

\section{B3. Futuro de Boi Gordo com Liquidação Financeira - Características}

Técnicas. Disponível em: <https://www.b3.com.br/pt br/produtos-eservicos/negociacao/commodities/ficha-do-produto8AE490CA6D41D4C7016D45F3CA183814.htm>. Acesso em: 05 jan. 2021.

CASILLO, A. Model specification for the estimation of the optimal hedge ratio with stock index futures: an application to the Italian derivatives market. In:

CONFERENCE ON DERIVATIVES AND FINANCIAL STABILITY, 1, 2004, Rome. Anais [...]. Rome, 2004.

COMEX STAT. Exportação e Importação Geral. Disponível em: $<$ http://comexstat.mdic.gov.br/pt/geral >. Acesso em: 09 jan 2021.

EDERINGTON, L. H. The Hedging Performance of the New Futures Markets. The Journal of Finance, v. 34, n. 1, p. 157-170, mar. 1979.

ENGLE, R. F.; GRANGER, C. W. Co-integration and Error Correction:

Representation, Estimation and Testing. Econometrica, v. 55, p. 251-276, 1987.

ENGLE, R. F.; KRONER, K. F. Multivariate Simultaneous Generalized GARCH.

Econometric Theory, v. 11, n. 1, p. 122-150, 1995.

FAMA, E. F. Efficient capital markets: a review of theory and empirical work. The Journal of Finance. New York. v. 25, n. 2, p. 383-417, mai. 1970.

FREITAS, C. A.; ALVES, W. B. Análise da Efetividade e da Razão Ótima do Hedge de Boi Gordo e do Cross-Hedge do Bezerro no Estado de São Paulo (2001 a 2010). Análise Econômica. Porto Alegre, v. 31, n. 60, p. 175-202, set. 2013.

GONÇALVES, D.; FRANCISCHINI, A.; ALVES, A.; PARRÉ, J. Análise de cointegração, causalidade e efetividade do hedge para os preços à vista e futuro do contrato de boi gordo para a região noroeste do Paraná. In: XLV Congresso da Sociedade Brasileira de Economia e Sociologia Rural, Anais [...]. Londrina, 2007.

HULL, J. C. Opções, Futuros e Outros Derivativos. 9. ed. Porto Alegre: Bookman, 2016. 
INSTITUTO BRASILEIRO DE GEOGRAFIA E ESTATÍSTICA - IBGE. Estatísticas. Disponível em: <https://www.ibge.gov.br/estatisticas/economicas/agricultura-epecuaria/9107-producao-da-pecuaria-municipal.html?edicao=29151\&t=sobre $>$. Acesso em: 07 jan. 2021.

INVESTING.COM. Boi Gordo Futuros Dados Históricos. Disponível em: $<$ https://br.investing.com/commodities/live-cattle-historical-data?cid=964528 >. Acesso em: 04 jan. 2021.

JOHNSON, L. L. The Theory of Hedging and Speculation in Commodity Futures. The Review of Economic Studies, v. 27, n. 3, p. 139-151, jun. 1960.

KUMAR, B.; SINGH, P.; PANDEY, A. Hedging effectiveness of constant and time varying hedge ratio in Indian stock and commodity futures markets.

Ahmedabad: Indian Institute Management, 2008. (Working Paper).

LAZZARINI, D. A. B. A taxa ótima de hedge no mercado brasileiro do boi gordo: uma abordagem com BEKK, DCC e BEKK com dummies de safra e entressafra. 2010. 41 f. Dissertação (Mestrado em Economia de Empresas) - Fundação Getúlio Vargas, São Paulo.

LIEN, D. D. The Effect of Cointegration Relationship on Future Hedging. Journal of Futures Markets, v. 16, n. 7, p. 773-780, 1996.

MADALA, G. S. Introdução à econometria. 3. ed. Rio de Janeiro: Livros Técnicos e Científicos, 2003.

MARKOWITZ, H. Portfolio Selection. The Journal of Finance, v. 7, n. 1, p. 77-91, 1952.

MYERS, R. J.; THOMPSON, S. R. Generalized Optimal Hedge Ratio Estimation. American Journal of Agricultural Economics, v. 71, n. 4, p. 858-868, nov. 1989.

RODRIGUES, M. A.; ALVES, A. F. Efetividade e razão ótima de hedge: um survey. In: 48 CONGRESSO DA SOCIEDADE BRASILEIRA DE ECONOMIA, ADMINISTRAÇÃO E SOCIOLOGIA RURAL, 48., 2010, Campo Grande. Anais [...]. Campo Grande: Sober, 2010.

TONIN, J. M.; BRAGA, M. J.; COELHO, A. B. Efetividade de hedge do milho com contratos futuros da BM\&F: uma aplicação para a região de Maringá, PR. Revista de Economia, Curitiba, v. 35, n. 31, p. 115-140, 2009.

UNITED STATES DEPARTMENT OF AGRICULTURE - USDA. Livestock and Poultry: World Markets and Trade. Disponível em: $<$ https://downloads.usda.library.cornell.edu/usdaesmis/files/73666448x/76537r01m/fx71bb52q/livestock poultry.pdf>. Acesso em: 16 jan. 2021. 


\section{APÊNDICE}

Figura A1 - Análise dos resíduos: $\Delta P_{v, t}=-0,71 Z_{t-1}-0,08 \Delta \ln P_{v, t-1}+0,06 \Delta \ln P_{f, t-1}$

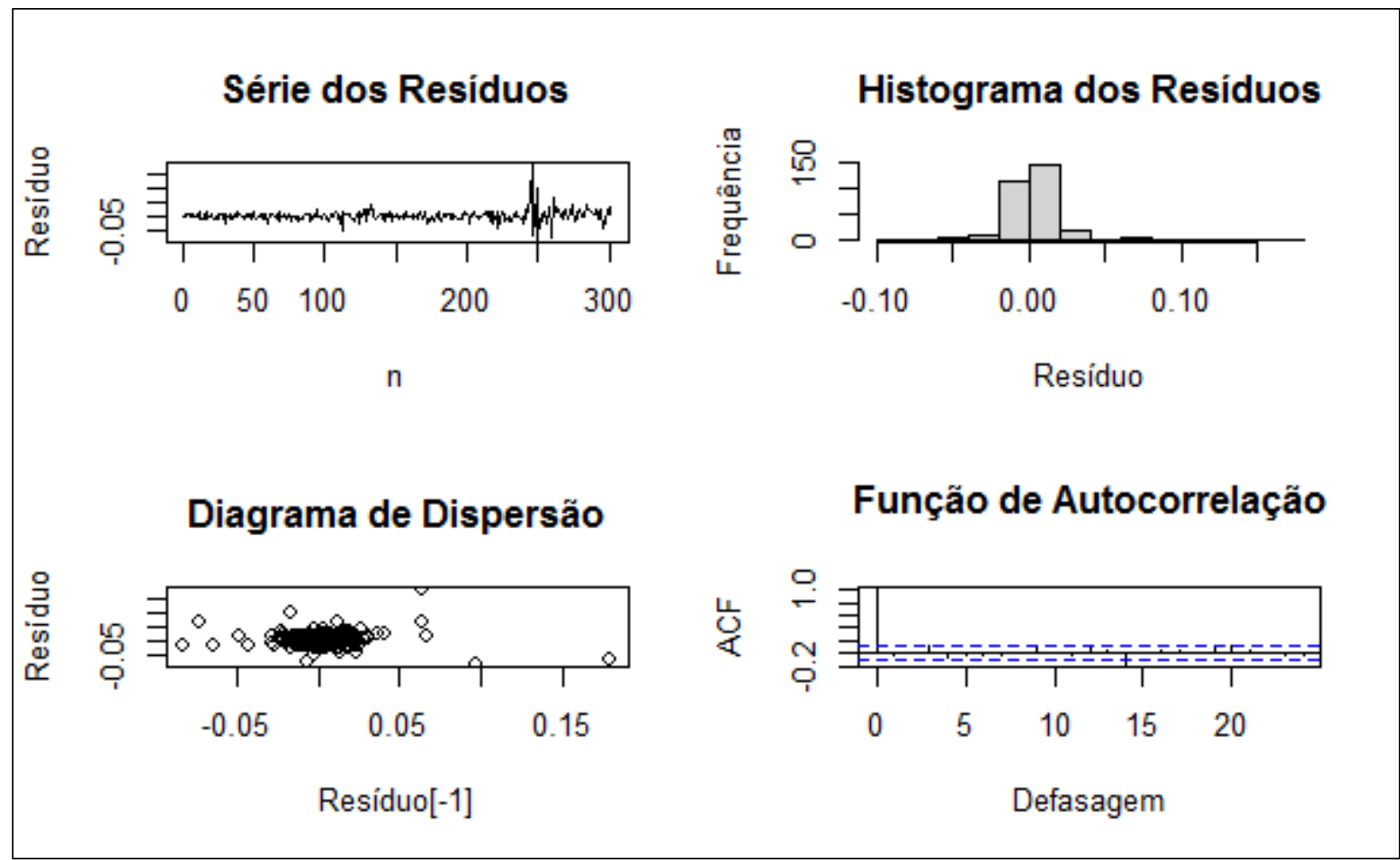

Fonte: Elaborado pelos autores (2021).

Figura A2 - Análise dos resíduos: $\Delta P_{f, t}=-0,12 Z_{t-1}+0,03 \Delta \ln P_{v, t-1}-0,04 \Delta \ln P_{f, t-1}$

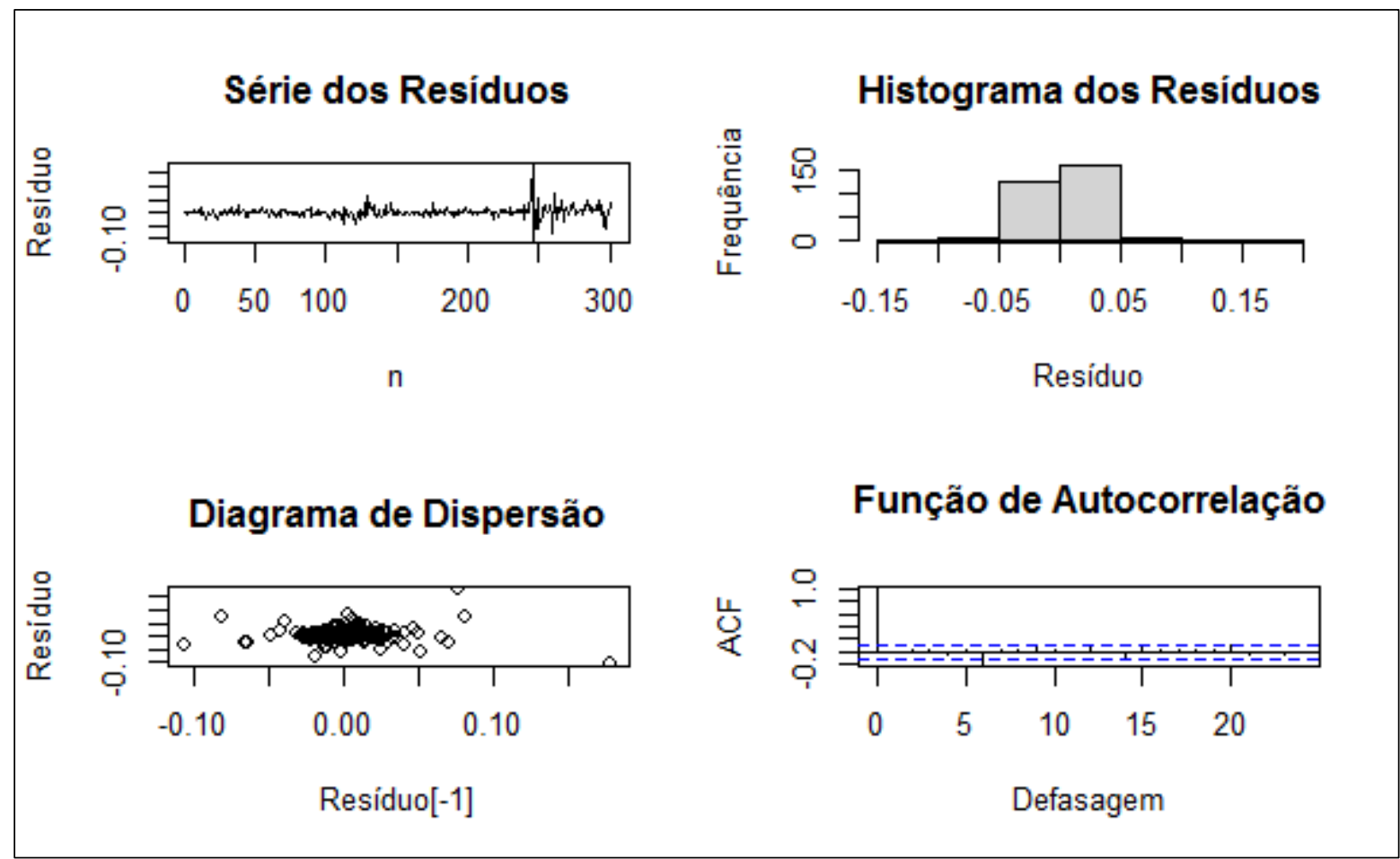

Fonte: Elaborado pelos autores (2021). 
Gestão e Desenvolvimento em Revista V. 8, N. 2, jul-dez/2021, p. 04-21.

ISSN online: $2446-8738$

Artigo recebido em: 28/10/2021

Artigo aprovado em: 28/11/2021

Figura A3 - Análise da presença de heterocedasticidade via Função de Autocorrelação (ACF).

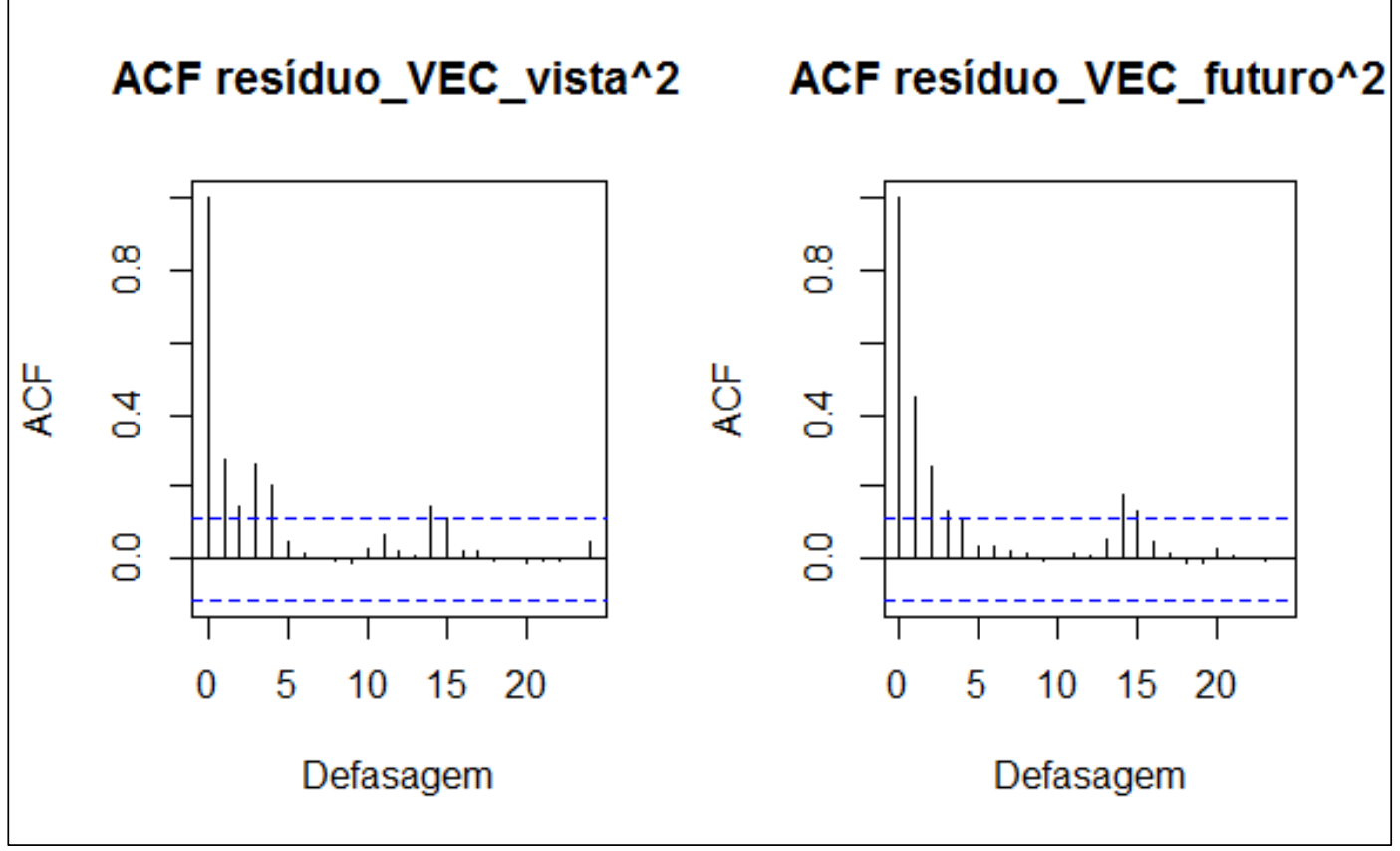

1. Fonte: Elaborado pe 